\title{
Avoiding Contrast-Enhanced Computed Tomography Scans in Patients With Shellfish Allergies
}

\author{
Anand K. Narayan, MD, PhD, Daniel J. Durand, MD¹, Leonard S. Feldman, MD²
}

${ }^{1}$ Department of Radiology, Johns Hopkins University School of Medicine, Baltimore, Maryland; ${ }^{2}$ Department of Medicine and Department of Pediatrics, Johns Hopkins University School of Medicine, Baltimore, Maryland.

The "Things We Do for No Reason" (TWDFNR) series reviews practices which have become common parts of hospital care but which may provide little value to our patients. Practices reviewed in the TWDFNR series do not represent "black and white" conclusions or clinical practice standards, but are meant as a starting place for research and active discussions among hospitalists and patients. We invite you to be part of that discussion.

A 55-year-old patient with a history of chronic obstructive pulmonary disease and diabetes mellitus presented to the emergency room with acute shortness of breath and right leg swelling that began 1 week after lumbar disk surgery. The emergency department team decides against ordering a chest CT scan with contrast to evaluate for a possible pulmonary embolism after noting that the patient's allergies include shellfish, which cause urticaria and facial edema. A ventilationperfusion scan reveals heterogeneous perfusion defects consistent with an intermediate probability $(20 \%-$ $80 \%$ ) for pulmonary embolism. The treating physicians consider starting the patient on a steroid regimen to prepare him for a CT scan with IV contrast, while presumptively anticoagulating the patient for 24 hours in order for the steroids to provide maximal protective effect before obtaining the scan. Should a history of shellfish allergy affect decision making regarding whether to administer IV contrast?

\section{WHY YOU MIGHT THINK ASKING ABOUT SHELLFISH ALLERGIES BEFORE PERFORMING CONTRAST-ENHANCED CT SCANS IS HELPFUL}

Fish and shellfish contain iodine, and allergic reactions to seafood are quite common, with a prevalence ranging anywhere between $2 \%$ and $6 \%$ of the population. ${ }^{1}$ As a result, patients with suspected shellfish allergies are often told by providers that they are allergic to iodine. In 1 study, nearly $92 \%$ of patients

*Address for correspondence and reprint requests: Anand Narayan, MD, Department of Radiology, Johns Hopkins University School of Medicine, 600 N. Wolfe St., Baltimore, MD 21287; Telephone: 410-955-6500; Fax: 410-550-1183; E-mail: anaraya1@jhmi.edu

Additional Supporting Information may be found in the online version of this article.

Received: July 28, 2015; Revised: October 5, 2015; Accepted: October 7,2015

2015 Society of Hospital Medicine DOI 10.1002/jhm.2509

Published online in Wiley Online Library (Wileyonlinelibrary.com). presenting to a pediatrics clinic with a suspected seafood or shellfish allergy cited iodine as the culprit. ${ }^{2}$ As contrast-enhanced CT scans utilize a variety of iodine-based agents, patients are often told to avoid CT scans with iodinated contrast agents or receive corticosteroid/antihistamine premedications prior to undergoing CT scans to mitigate potentially lifethreatening allergic reactions. A survey of radiologists and interventional cardiologists revealed that $65.3 \%$ and $88.9 \%$, respectively, asked about seafood or shellfish allergies prior to administering contrast enhanced CT scans, and $34.7 \%$ and $50.0 \%$, respectively, stated that they would withhold contrast media or recommend premedication with corticosteroid/antihistamines for patients with seafood or shellfish allergy. ${ }^{2}$

\section{WHY ASKING ABOUT SHELLFISH ALLERGIES BEFORE IV CONTRAST CT SCANS DOES NOT REDUCE THE RISK OF CONTRAST REACTIONS}

\section{What Causes Allergic-Like Reactions to Fish and Shellfish?}

Allergic reactions are inappropriate or exaggerated immune response (hypersensitivity reaction). Four types of hypersensitivity reactions have been described (type I-IV $)^{3}$; allergic reactions mediated by immunoglobulin $\mathrm{E}(\mathrm{IgE})$ represent type I hypersensitivity reactions.

Although fish and shellfish contain iodine, so too do a wide variety of commonly consumed foods (eg, yogurt, milk, bread). In addition, our bodies contain and require sufficient quantities of iodine for basic functions, making immune reactions to such an essential ingredient of life unlikely. Instead, fish and shellfish contain proteins (parvalbumin and tropomyosins, respectively), which act as the major allergens, not iodine. $^{4}$

\section{What Causes Reactions to IV Contrast Media?}

Around the world, tens of millions of injections occur every year for contrast-enhanced scans. ${ }^{5}$ Reactions to IV contrast media are not uncommon, occurring anywhere between $0.6 \%$ and $17 \%$ of the time, with severe reactions occurring between $0.02 \%$ and $0.5 \%$ of the time. ${ }^{6}$ Higher reaction rates were associated with the use of higher-osmolarity contrast agents. A review of research studies found a lower rate of reactions to IV contrast in eras in which low-osmolarity 
agents were exclusively used $(0.2 \%$ after 1991$)$ versus eras in which high-osmolarity agents were exclusively used $\left(7.0 \%\right.$ between 1985 and 1986). ${ }^{7}$

Reactions to contrast include allergic-like reactions as well as a variety of other reactions (eg, arrhythmias, vasovagal reactions, flushing), which are thought to be related to the dose and concentration of contrast media. ${ }^{8}$

Allergic-like, or anaphylactoid, reactions related to contrast are largely thought to have a fundamentally different molecular mechanism than true classic allergic reactions. Anaphylactoid reactions are caused by direct release of histamine into the bloodstream in response to interacting with chemicals. These reactions are not related to or mediated by IgE antibodies and do not require prior exposure.

True classic allergic reactions, on the other hand, are mediated by IgE antibodies in which initial exposure to an allergen (antigen) is followed by subsequent exposure and production of IgE antibodies. ${ }^{9}$ The allergen-IgE antibody complex causes the degranulation of mast cells and basophils, leading to the release of histamines.

Reactions to IV contrast are likely related to some component of the contrast media instead of the iodine itself. It is thought that the majority of these reactions are anaphylactoid reactions instead of true classic allergic reactions, given that $\mathrm{IgE}$ antibodies are not consistently elevated in patients who exhibit these reactions. ${ }^{8}$ Nevertheless, the symptoms of these 2 types of reactions (anaphylactoid and allergic reactions) are similar and require comparable treatment to prevent life-threatening anaphylaxis.

\section{What Are the Major Risk Factors for Allergic-Like Contrast Reactions?}

Previous studies on risk factors for allergic-like contrast reactions suggest that the strongest predictor of future contrast reactions is a history of prior contrast reaction (5-fold higher risk), with an estimated $10 \%$ to $35 \%$ recurrence risk of contrast reactions. ${ }^{8}$ Patients with a history of atopy, asthma, and food allergies (including seafood) are at approximately 2 to 3 times greater risk of contrast reactions. ${ }^{9}$

\section{Do Shellfish Allergies Place Patients at Higher Risk for Contrast Reactions Than Other Allergies?}

In 1 of the few studies evaluating seafood allergies specifically, Witten et al. compared the frequency of contrast reactions in patients with histories of seafood allergy, food allergy, asthma, hay fever, hives, and contrast medium. ${ }^{10}$ Using their results, we compared the frequency of reactions in patients with histories of seafood allergy $(6.3 \%, 4 / 64)$ to patients with any other type of allergy or atopic state $(9.2 \%, 212 / 2304)$ and found no statistically significant differences $(P=$ 0.418). Similarly, Shehadi evaluated seafood as well as asthma, hay fever, common medications (eg, penicillin, aspirin, morphine), and others. ${ }^{11} \mathrm{~A}$ reanalysis of the results found no statistically significant differences comparing the frequencies of contrast reactions in patients with seafood allergy $(15.0 \%)$ compared with other allergens (eggs, milk and chocolate, $14.6 \%$; general allergies, $13.1 \%$; fruit allergies, $12.9 \%$; asthma, $11.2 \% ; P$ values ranging between 0.2 and 0.6$).{ }^{6}$ Overall, the results suggest that patients with seafood allergy are at no higher risk for having a contrast reaction compared with patients with other food allergies or other forms of atopy.

Additionally, seafood and other food allergies should be distinguished from food intolerances in which the ingestion of histamine-rich materials in conjunction with histamine inhibitors (drugs or alcohol) leads to symptoms that can mimic allergic-like reactions (urticaria, pruritus, diarrhea, asthma). ${ }^{12}$

\section{What Do the Guidelines Recommend?}

For patients who require IV contrast media for CT scans, the American Academy of Allergy, Asthma, and Immunology recommends not routinely ordering lowiso-osmolar radiocontrast media or pretreating with corticosteroids and antihistamines for patients with a history of seafood allergy. ${ }^{13}$ The American College of Radiology recommends pretreatment with corticosteroids only for those patients who have previously experienced moderate to severe reactions to IV contrast. ${ }^{8}$

\section{WHAT YOU SHOULD DO INSTEAD: ASK ABOUT PRIOR CONTRAST REACTIONS BEFORE ADMINISTERING CONTRAST}

When a patient presents for a contrast-enhanced CT scan, patients should be asked if they have experienced reactions to contrast and the severity and type of the associated reactions. Providers and support staff should not ask specifically about shellfish allergies, as they have not been found to be associated with an elevated risk of contrast reactions compared with other allergens. Although all allergies seem to increase the likelihood of having a reaction to contrast, only a history of previous contrast reactions will prompt a change in management. Asking specifically about seafood allergies before performing an IV contrast CT scan is a Thing We Do for No Reason.

\section{RECOMMENDATIONS}

1. Before performing contrast-enhanced CT scans, patients should be asked if they have experienced reactions to IV contrast. There is no reason for providers and support staff to specifically inquire about seafood allergies.

2. Patients with seafood and other food allergies do not require premedication prior to CT scans. Seafood and other food allergies do not represent contraindications to obtaining contrast-enhanced CT scans and should not prompt a change in management. 
Disclosures: The authors do not have any relevant financial disclosures to report.

Do you think this is a low-value practice? Is this truly a "Thing We Do for No Reason"? Share what you do in your practice and join in the conversation online by retweeting it on Twitter (\#TWDFNR) and liking It on Facebook. We invite you to propose ideas for other "Things We Do for No Reason" topics by emailing TWDFNR@hospitalmedicine.org

\section{References}

1. Hajeb P, Selamat J. A contemporary review of seafood allergy. Clin Rev Allergy Immunol. 2012;42(3):365-385.

2. Beaty AD, Lieberman PL, Slavin RG. Seafood allergy and radiocontrast media: are physicians propagating a myth? Am J Med. 2008;121: 158:e1-e4.

3. Riedl MA, Casillas AM. Adverse drug reactions: types and treatment options. Am Fam Physician. 2003;68(9):1781-1790.

4. Leung PS, Chen YC, Chu KH. Seafood allergy: tropomyosins and beyond. I Microbiol Immunol Infect. 1999;32(3):143-154.

5. Pasternak JJ, Williamson EE. Clinical pharmacology, uses, and adverse reactions of iodinated contrast agents: a primer for the nonradiologist. Mayo Clin Proc. 2012;87(4):390-402.

6. Schabelman E, Witting M. The relationship of radiocontrast, iodine, and seafood allergies: a medical myth exposed. J Emerg Med. 2010; 39(5):701-707.
7. Cochran ST, Bomyea K, Sayre JW. Trends in adverse events after IV administration of contrast media. AJR Am J Roentgenol. 2001; 176(6):1385-1388.

8. ACR Manual on Contrast Media. Version 9. Reston, VA: American College of Radiology, ACR Committee on Drugs and Contrast Media; 2013.

9. Coakley FV, Panicek DM. Iodine allergy: an oyster without a pearl? AJR Am J Roentgenol. 1997;169(4):951-952.

10. Witten DM, Hirsch FD, Hartman GW. Acute reactions to urographic contrast medium: incidence, clinical characteristics and relationship to history of hypersensitivity states. Am J Roentgenol Radium Ther Nucl Med. 1973;119(4):832-840.

11. Shehadi WH. Adverse reactions to intravascularly administered contrast media. A comprehensive study based on a prospective survey. Am J Roentgenol Radium Ther Nucl Med. 1975;124: $145-152$.

12. Boehm I. Seafood allergy and radiocontrast media: are physicians propagating a myth? Am J Med. 2008;121(8):e19.

13. Choosing Wisely. American Academy of Allergy, Asthma \& Immunology. Don't routinely order low- or iso-osmolar radiocontrast media or pretreat with corticosteroids and antihistamines for patients with a history of seafood allergy, who require radiocontrast media. Available at: http://www.choosingwisely.org/clinician-lists/ american-academy-allergy-asthma-immunology-low-or-iso-osmolarradiocontrast-media/. Published March 3, 2014. Accessed July 12, 2015 . 